\title{
MEASUREMENT AND SIMULATION OF THE UMER BEAM IN THE SOURCE REGION*
}

\author{
I. Haber, S. Bernal, R. A. Kishek, P. G. O’Shea, B. Quinn, M. Reiser, Y. Zou \\ University of Maryland, College Park, MD 20742-3511 \\ A. Friedman, D. P. Grote, J. -L. Vay \\ LBNL 1 Cyclotron Road Bldg 47 Berkeley, CA, 94720-8201
}

\begin{abstract}
As the beam propagates in the University of Maryland Electron Ring (UMER) complex transverse density structure including halos has been observed. A primary objective of the experiment is to understand the evolution of a space-charge-dominated beam as it propagates over a substantial distance. It is therefore important to understand which details of the beam structure result from propagation of the beam in the ring and which characteristics result from the specific details of the initial distribution. Detailed measurements of the initial beam characteristics have therefore been performed. These include direct measurement of the density using a phosphor screen, as well as pepper pot measurements of the initial transverse distribution function. Detailed measurements of the distribution function have also been obtained by scanning a pinhole aperture across a beam diameter, and recording phosphor screen pictures of the beam downstream of the pinhole.

Simulations of the beam characteristics in the gun region have also been performed using the WARP P.I.C. code. From these simulations, the observed behavior has been attributed to a combination of perturbations to the transverse distribution by a cathode grid that is used to modulate the beam current, as well as the complex transverse dynamics that results from the combination of the nonlinear external focusing fields of the gun structure and the nonlinear space charge forces.
\end{abstract}

\section{INTRODUCTION}

Even though many of the basic characteristics of space-charge-limited diodes have been well known for several decades, this knowledge does not usually extend to the details of the particle distribution function that are necessary for predicting the downstream behavior of modern intense-beam systems. Furthermore, recent simulations and experiments have found that details of the beam distribution emerging from the source can have a strong influence on the downstream beam evolution. Since the phenomena that govern the beam evolution are usually nonlinear and measurements of the beam emerging from the gun region with adequate accuracy are often quite difficult, simulations have become a promising tool for understanding the behavior of space-chargedominated beams in the source region. However, an important precursor to developing a credible predictive simulation capability that can reliably model the source region is the benchmarking of the numerics against experimental observation.

An effort has therefore been undertaken to compare numerical simulation against experiment in those cases where beam diagnostics are sufficient to permit accurate simulation/experiment comparisons for spacecharge-dominated sources. It should be noted that even though this work has largely been motivated by developing a predictive capability for the highly space-charge-dominated beams required for heavy ion fusion, the results are applicable to a large class of machines. This is because as the intensity or luminosity of a beam at the high-energy end of an accelerator is increased, it becomes increasingly important to operate the source region in the space-chargedominated regime.

Recent research comparing WARP simulations to experimental observation of gun behavior have centered on two experiments that are particularly suitable for these comparisons because of the diagnostic configurations.

* This work is supported by the US DOE under contract Nos. DE-FG02-02ER54672 and DE-FG02-94ER40855 (UMD), and DE-AC03-76SF00098 (LBNL) and W-7405-ENG-48 (LLNL). ｅmail: haber@umd.edu 
These are the $500 \mathrm{KeV}$ Source Test Stand (STS-500) diode at LLNL, and the University of Maryland Electron Ring (UMER). However, because the work on the STS-500 is described elsewhere,[1] the description here will emphasize simulation/experiment comparisons on UMER.

\section{THE UMER GUN}

The diode in the UMER experiment is a simple Pierce geometry but with two significant modifications. To ensure an equipotential surface across the anode plane, an anode grid with approximately $86 \%$ transparency has been placed across the anode aperture. A more significant modification of the gun characteristics results from a cathode grid with $0.0254 \mathrm{~mm}$ diameter wires $0.15 \mathrm{~mm}$ apart, in a window-screen pattern, placed $0.15 \mathrm{~mm}$ from the cathode surface. The presence of this grid creates a triode geometry that is used to modulate the current by varying the applied grid-to-cathode voltage. In the normal operating range, the cathode-to-grid potential is set so the triode operates in a saturated state to avoid amplification of any fluctuations on the grid pulse. A cartoon representation of the gun geometry, as well as the aperture-wheel /phosphor-screen combination is shown in Fig. 1. As will be discussed below, operating with a grid-to-cathode potential sufficient to saturate the triode significantly modifies the transverse velocity distribution from what is obtained in a simple diode. Furthermore, this modification of the transverse distribution was found to have a significant effect on the downstream evolution. In fact, it does not appear possible to accurately model the downstream behavior without including the modification to the beam velocity distribution by the cathode grid.

\section{EXPERIMENTAL MEASUREMENTS}

The primary source of experimental data on the detailed characteristic of the beam emerging from the gun has come from images on the phosphor screens that can be inserted into the beam. One of these screens is placed approximately $28.8 \mathrm{~cm}$ downstream from an aperture wheel that is approximately $20 \mathrm{~mm}$ from the anode plane and can be lowered into the beam line without breaking vacuum. This screen, used along with a pinhole aperture that can be scanned across the beam diameter, is used to measure the velocity distribution across the beam, as well as the transverse variation in the current density. If the beam is assumed axisymmetric, a full transverse phase-space distribution is obtained. Another screen, placed on a long plunger-like mechanism that allows the screen to be slid along the injector line, cannot be used if any of the sections of the ring are in place. Data from the movable screen is, therefore, limited to what was obtained during the commissioning of the injector line.

Figure 2 is an image, under typical operating conditions, of the fixed position phosphor screen $28.8 \mathrm{~cm}$ downstream of the aperture wheel when the pinhole aperture is positioned at the beam center. The DC bias voltage applied to the cathode-grid gap, which acts to impede emission from the cathode in the absence of the beam pulse, is set at approximately $-40 \mathrm{~V}$. During the nominal 100 ns duration of the approximately $70 \mathrm{~V}$ positive pulse applied to the grid, the grid-to-cathode potential is estimated to be about $30 \mathrm{~V}$, which is the sum of the bias voltage and applied pulse voltage of opposite polarity. (The actual voltage is not precisely known because the magnitude of the voltage drop, as the grid draws current during the pulse, is difficult to estimate.)

The length limitation does not permit a comprehensive discussion of the measurements that have been performed, however, two surprising features to note are the observed shape of the velocity distribution, which can be described as hollowed and axisymmetric, and the transverse uniformity of this hollowed velocity distribution which is found to be approximately the same across the beam cross section. This transverse uniformity of the distribution was also seen, but in less detail, in the previously published pepper-pot phosphor screen images [2] obtained in an earlier experiment.

Figure 3 is a plot of the transverse density variation measured, using the moveable phosphor screen placed near the anode plane, during commissioning of the injector line. The curve was obtained by plotting the intensity of the image along a diagonal cut across through the beam. The data presented here is only a small but representative sample of the data taken over a variety of gun operating conditions. However, it is still sufficient to illustrate some of the important characteristics of the UMER gun. 


\section{SIMULATIONS OF THE UMER GUN}

Because of the disparity in scale between the $0.15 \mathrm{~mm}$ distance between grid wires and the $0.15 \mathrm{~mm}$ distance of these wires from the cathode surface on the one hand, and the approximately $25 \mathrm{~mm}$ distance to the anode, (this distance can be varied to adjust the gun perveance) it is difficult to simulate all the characteristics of the gun simultaneously. A two-pronged approach was therefore adopted that first examined the macroscopic characteristics of the gun structure by legislating the distribution function of the current emerging from the grid. The grid-tocathode physics responsible for the experimentally observed distribution, especially the hollowed velocity space, were then examined in the second (and still continuing) phase.

Some of the results of the macroscopic simulations of the full diode gemoetry have already been described.[2] Axisymmetric WARP simulations were performed assuming a transversely uniform current density injected at the plane of the cathode grid. The magnitude of this current was chosen to match the measured total current after accounting for the fraction intercepted by the anode grid. A series of simulations was performed to examine sensitivity of the simulated profile at the plane of the phosphor screen to the assumed form of the injected velocity distribution. This profile was found to be surprisingly sensitive to the assumed shape of the initial velocity distribution. This sensitivity was unanticipated because it was assumed that, as a result of the dominance of space charge potential energy over the transverse kinetic energy in the beam, the measured profile should be relatively insensitive to the details of the initial velocity distribution. An additional surprise, discussed elsewhere [3] is the degree to which the downstream behavior as the beam propagates in the ring also depends on the detailed form of the initial velocity distribution.

Figure 4 is a plot of the radial density variation of the simulated distribution propagated to plane of the phosphor screen. Note that since the simulation is axisymmetric, only half of the distribution from the center to the outer edge is plotted, in contrast to the density plot in Fig. 3 in which both halves of the measured beam are plotted to explicitly exhibit the degree of asymmetry in the experiment. By varying the injected velocity distribution to obtain qualitative agreement with the measured transverse variation in the current density, a surprising degree of detailed agreement was observed to what were initially thought to be measurement anomalies. Because these features, such as the dip in density at the beam center and the small shoulder out from the center, appear in both the simulated and measured density they appear to be real, if not completely understood, features of the beam evolution. It should be noted that such agreement is absent if the initial distribution is varied significantly from the assumed form.

Although the shape of the central density dip in the simulation differs from the measured central dip, from the left to right asymmetry in the experimental curve shown in Fig. 3 it seems clear that the depression in the measured distribution is not centered on the diameter cut across the beam that was used to generate the plot. This occurs because the density depression near the center of the beam is slightly displaced. This displacement is thought to result from a slight misalignment of the gun as well as the possibility that the simulation geometry does not precisely mirror the "as-constructed" gun geometry. In addition, the sharpness in the dip in the simulated curve in Fig. 4 might be exaggerated by slight numerical inaccuracy in the simulation at the beam center. An additional source of potential disagreement between simulation and measurement is the simplified, two-parameter fit for the initial velocity distribution, which is characterized by oppositely-directed Gaussian distributions of specified width and separation. Similar comments apply to the slight shoulders in the density curves, seen in both the simulation and experiment, as well as the slight skirt at the beam edge. While the shoulders in the two curves do not correspond precisely in location or shape, such shoulders are a feature both in simulations and measurement over a range of parameters.

The dips, as well as the shoulders, were unexpected because the normalized beam emittance in the simulation of $12.5 \square \mathrm{m}$ is only about $2.5 \square$ the intrinsic emittance calculated from the product of the $0.1 \mathrm{eV}$ cathode temperature and the emitter radius. This led to an expectation of a laminarity for the particle orbits that was not expected to produce such structure.

The simulations of the macroscopic gun characteristics were performed assuming an 
initial hollowed distribution. Simulations have also been undertaken to understand the mechanisms that can produce this hollowing. Three-dimensional simulations that resolve the very fine scale of the cathode grid at the same time as simulating the much larger diode as a whole are an ambitious undertaking. Instead, three-dimensional WARP simulations were undertaken that examine a single $0.15 \mathrm{~mm}$ square cell in the periodic array of grid cells near the beam center. Periodic boundaries, as well as fourfold transverse symmetry, were assumed in the simulation so that the model does not include any transverse influence associated with the evolution of the beam edge or with any beam compression from the transverse focusing applied by the Pierce geometry.

Because the dynamics of virtual cathode formation near the emitter surface and downstream of the grid are important to the resulting distribution [4] the cathode was modeled by injecting several times the expected Child- Langmuir current from the cathode surface and following the self consistent temporal evolution as a virtual cathode is formed. It should be noted that the finest grid used to model the $0.15 \mathrm{~mm}$ square region 25 $\mathrm{mm}$ long, with the assumption of fourfold transverse symmetry in the two transverse dimensions, was 2048 by 32 by 32 . This grid was not completely adequate to resolve the longitudinal dynamics so that some possibly nonphysical transient behavior [5] was observed. Nevertheless, though refinement of the simulations is currently underway, the qualitative features observed are believed to be correct, particularly because of the extent that they mirror experimental observation.

What is observed in the simulations is that for small grid to cathode potentials, the virtual cathode forms between the cathode and grid, and observed downstream transverse velocity distribution function is monotonically decreasing with velocity and some observed squaring of the distribution, as opposed to the axisymmetry expected without the grid, matches experimental observation. However, above a threshold in the magnitude of the gridto-cathode voltage of approximately $30 \mathrm{~V}$, a virtual cathode is formed downstream of the cathode grid and the experimentally observed hollowing in the transverse velocity distribution is seen in the simulations. Figure 4 is a particle scatter plot of the downstream velocity distribution obtained from one such a simulation. While the current simulations are not fully converged numerically, and further refinement is in process, nevertheless the qualitative features of the experiment have been reproduced.

\section{CONCLUSIONS}

Both experimental observation and simulation have revealed a significant level of, unexpected, complexity in the evolution of the details of the beam distribution function as the beam traverses the diode region. For a sufficient grid-to-cathode potential difference a hollowing is observed in the transverse velocity distribution.

Though the hollowed velocity distribution does not appear to cause a large increase in the transverse beam emittance, some of the transverse structure observed during beam propagation in the diode region appears to deviate from what would be expected using the laminar orbit structure expected in a the highly space-charge-dominated region that is characteristic of a low emittance gun structure.

The agreement that has been seen between simulation and observation promotes substantial confidence in using the simulations as a tool for understanding the significant features of the space-charge physics. Also important, the detailed characterization of the beam distribution function of the beam that has been obtained by moving a small aperture across the beam should provide, along with the simulations, a valuable tool in predicting the detailed evolution of the beam propagation in the UMER ring.

\section{REFERENCES}

[[1] J. W. Kwan, F. M. Bieniosek, W.L. Waldron, J-L. Vay, G.A. Westenskow, E. Halaxa, I. Haber, "Production of a High Brightness Beam from a Large Surface Source," HIF2004

[[2] I. Haber, S. Bernal, C. M. Celata, A. Friedman, D. P. Grote, R. A. Kishek, B. Quinn, P. G. O'Shea M. Reiser, J-L. Vay, "Collective Space-Charge Phenomena in the Source Region," Nucl. Instr. and Methods A 519 (2004) 396. 
[3] R.A. Kishek, S. Bernal, C.L. Bohn, D. Grote, I. Haber, H. Li, P.G. O'Shea, M. Reiser, and M. Walter, "Simulation and experiments with space-charge-dominated beams," Physics of Plasmas 10 (2003) 2016.

[4] Y. Zou, H. Li, M. Reiser, and P.G. O'Shea, "Theoretical Study of Transverse Emittance Growth in a Gridded Electron Gun," Nucl. Instr. and Methods A 519 (2004) 432.
[5] J-L. Vay, P. Colella, J.W. Kwan P. McCorquodale, D.B. Serifini, A. Friedman, D.P. Grote, G. Westenskow, J.-C. Adam, A. Heron, and I. Haber, "Application of Adaptive Mesh Refinement to Particle-in-Cell Simulations of Plasmas and Beams," Physics of Plasmas 11 (2004) 2928. 


\section{FIGURE CAPTIONS}

Fig. 1. Schematic representation of the UMER gun geometry illustrating the presence of cathode grid (G) close to the emitter surface (K), as well as an anode grid (A) across the gun exit that is used to insure an equipotential at the anode plane. A wheel-mounted pinhole aperture (W) slightly downstream from the anode plane can be rotated across the beam to measure the velocity distribution across the beam by capturing the angular beam spread on a phosphor screen (S) $28.8 \mathrm{~cm}$ downstream.

Fig. 2. Phosphor screen image $28.8 \mathrm{~cm}$ downstream of a $0.5 \mathrm{~mm}$ pinhole at the beam center showing the hollowed transverse velocity distribution that can be caused by the cathode grid when the grids to cathode potential is sufficiently high. The calibration arrow is transverse distance on the image divided by the distance to the pinhole. The faint circle at the outside of the picture is the outer edge of the $3.175 \mathrm{~cm}$ diameter phosphor.

Fig. 3. Plot of intensity across a diameter taken from a phosphor screen image of the full beam $6 \mathrm{~cm}$ downstream of the anode plane showing the radial current density variation across the beam.

Fig. 4 Plot of the radial density variation from a WARP simulation of gun where a specified current matching the measured total current is inject at the cathode grid plane with a specified hollow transverse velocity distribution. The velocity distribution consists of transversely counterstreaming Gaussian distributions with a thermal spread corresponding to $0.2 \mathrm{eV}$ temperature and separated by 4 times that thermal spread.

Fig.5. Particle plot of the velocity space from a WARP simulation of a small region in the beam center corresponding to single cell of the periodic rectangular cathode grid, with fourfold transverse symmetry and periodic transverse boundary conditions. When a sufficiently large, in this case $30 \mathrm{~V}$, potential is applied between the grid and the cathode a virtual cathode is formed downstream of the grid and a hollowed velocity distribution similar to what is seen experimentally is observed. 



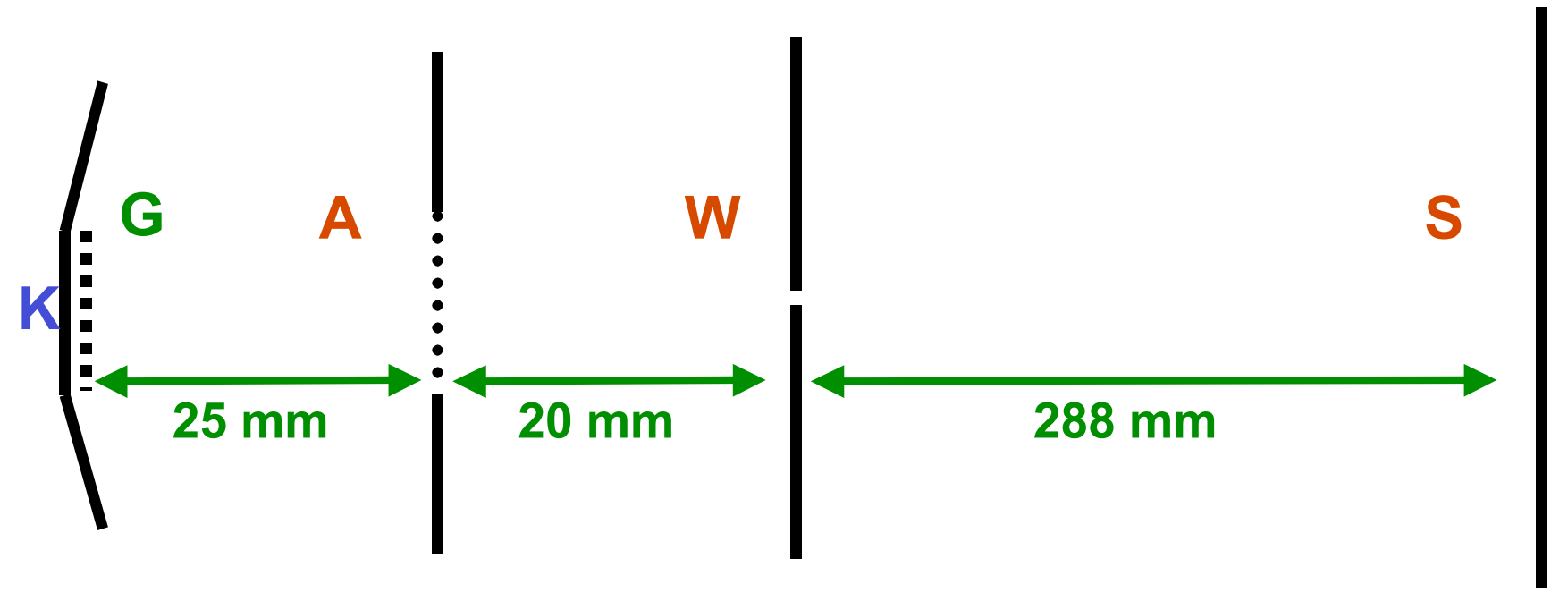

Fig. 1 
$\longrightarrow x \square=0.02$

Fig. 2 


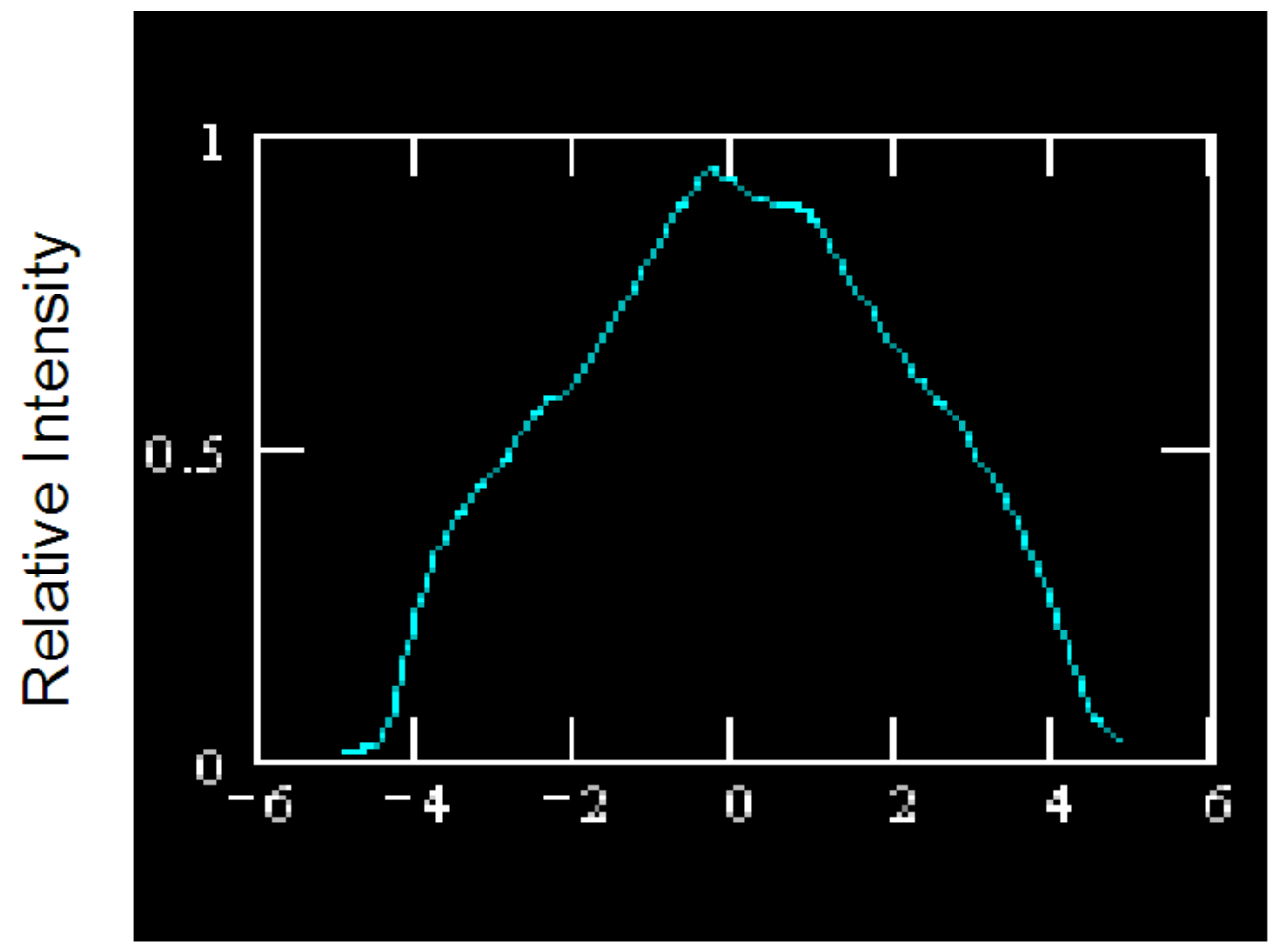

Distance across beam (mm)

Fig. 3 


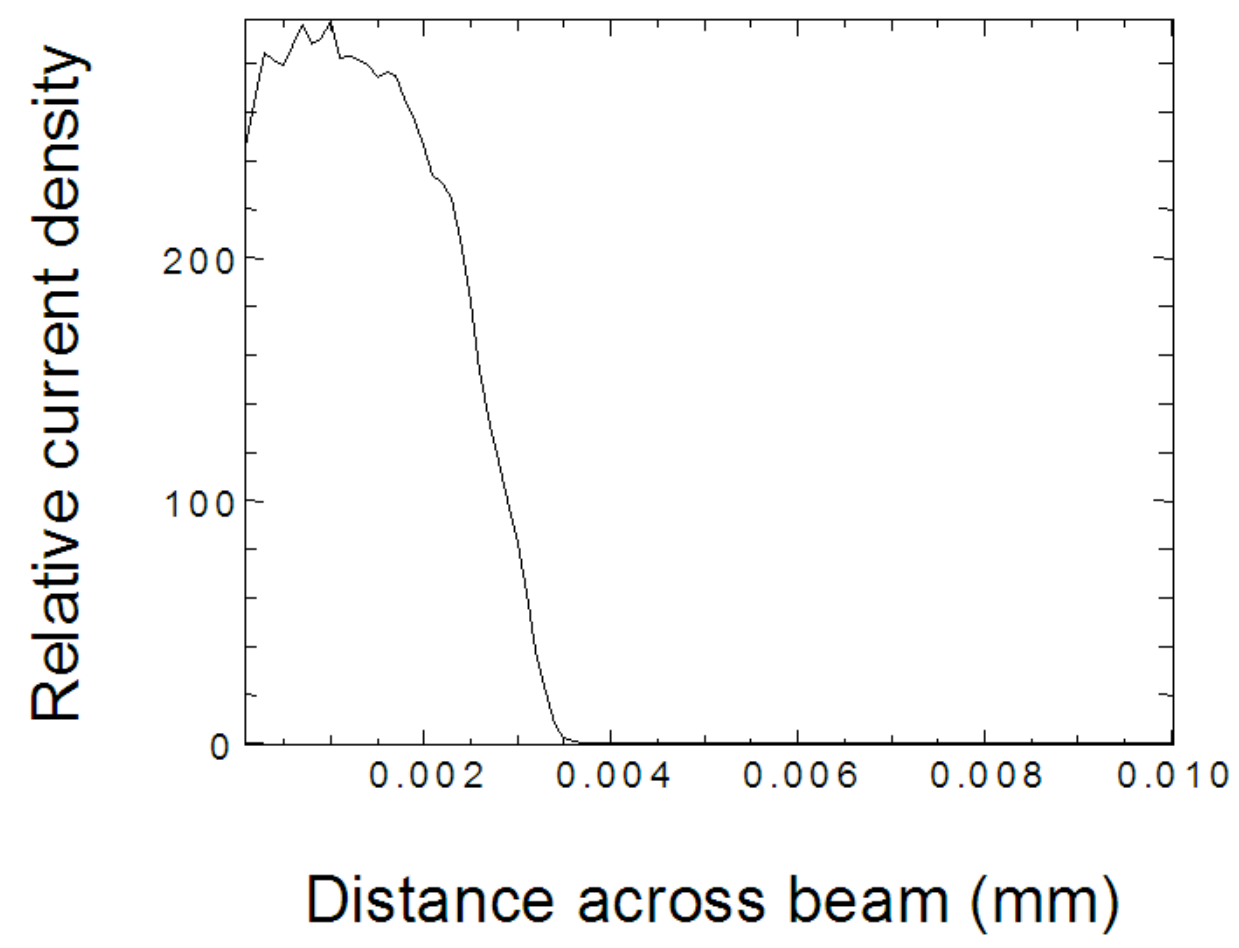

Fig. 4 


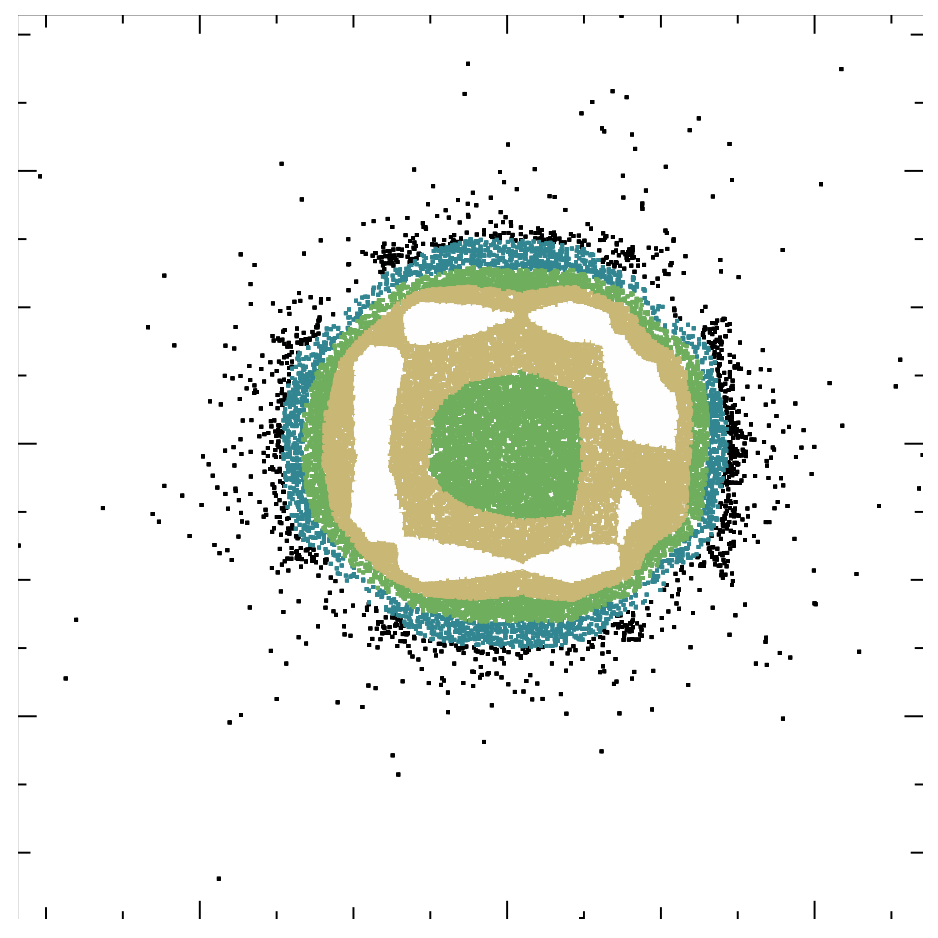

Fig. 5 